\title{
Low Complexity Approximate Log-MAP Detection for MIMO Systems.
}

\author{
Jos Akhtman and Lajos Hanzo \\ School of ECS., Univ. of Southampton, SO17 1BJ, UK. \\ Tel: +44-23-80-593 125, Fax: +44-23-80-593 045 \\ Email: 1h@ecs.soton.ac.uk, http://www-mobile.ecs.soton.ac.uk
}

\begin{abstract}
In this paper we propose a novel low-complexity Log-MAP space-time detection method, which can be regarded as an advanced extension of the Complex Sphere Decoder (CSD). We demonstrate that as opposed to the previously published variants of the soft-output CSD, the proposed technique may be employed in the so-called "over-loaded" scenario, where the number of transmit antennas exceeds that of the receive antennas. The proposed method closely approaches the optimum performance of the Log-MAP detector even in heavily over-loaded scenarios, while the associated computational complexity is only moderately increased.
\end{abstract}

\section{INTRODUCTION}

Multi-Carrier (MC) modulation techniques [1,2] have found their way into several wireless broadband communications standards as well as into local area networks. Owing to their advantageous properties they also constitute strong contenders for the next-generation cellular mobile communications standards.

The relevant information-theoretical analysis predicts [3] that substantial capacity gains are achievable in wireless communication systems employing a Multiple Input Multiple Output (MIMO) architecture using multiple antennas. Specifically, provided that the fading processes corresponding to different transmit-receive antenna pairs may be assumed to be independently Rayleigh distributed, the associated attainable capacity was shown to linearly increase with the smaller of the numbers of the transmit and receive antennas. Additionally, the employment of a MIMO architecture allows for the efficient exploitation of the spatial diversity available in a wireless MIMO environment, thus an improvement of the system's transmission integrity, as well as a further increase in the system's capacity becomes possible.

In [4] we have introduced a novel Optimized Hierarchy Reduced Search Algorithm (OHRSA)-aided space-time processing method, which may be regarded as an advanced extension of the Complex Sphere Decoder (CSD) method, portrayed in [6]. The algorithm proposed in [4] extends the potential application range of the CSD methods of [5] and [6], as well as reduces the associated computational complexity. Moreover, the OHRSAaided SDM detector proposed combines the near-optimum performance of the ML SDM detector with the low-complexity of the linear MMSE SDM detector, which renders it an attractive design alternative for practical systems.

It is widely recognized [1] that the BER associated with the

Acknowledgements: The work reported in this paper has formed part of the Wireless Enabling Techniques work area of the Core 3 Research Programme of the Virtual Centre of Excellence in Mobile and Personal Communications, Mobile VCE, www.mobilevce.com, whose funding support, including that of EPSRC, is gratefully acknowledged. Fully detailed technical reports on this research are available to Industrial Members of Mobile VCE. process of communicating over a noisy fading MIMO channel can be dramatically reduced by means of employing channel coding. A particularly effective channel coding scheme is constituted by soft-input soft-output turbo codes [8]. Turbo coding, however, requires soft information concerning the bit decisions at the output of the SDM detector, in other words the a posteriori soft information regarding the confidence of the bit-decision is required.

Consequently, in Section II we deduce the OHRSA-aided Log-MAP SDM detector, which allows for an efficient evaluation of the soft-bit information and therefore results in highly efficient turbo decoding. Unfortunately however, in comparison to the OHRSA-aided ML SDM detector derived in [4] the OHRSA-aided Log-MAP SDM detector of Section II exhibits a substantially higher complexity. Consequently, in Section III we derive an approximate Log-MAP method, namely the SOPHIE SDM detector. The SOPHIE SDM detector combines the low complexity of the OHRSA-aided ML detector described in [4] with the near-optimum performance of the OHRSA-aided Log-MAP SDM detector of Sections II. Specifically, it exhibits a similar performance to that of the optimal Log-MAP detector, while imposing a modest complexity, which in the scenario of employing QPSK is only slightly higher than that required by the low-complexity MMSE SDM detector [1]. The computational complexity as well as the achievable performance of the SOPHIE SDM detector of Section III are analysed and quantified in Section IV. Finally, our conclusions are summarized in Section V.

\section{OHRSA-AIDED LOG-MAP SDM DETECTION}

The subcarrier-related MIMO-OFDM system model considered is given by [1]

$$
\mathbf{y}=\mathbf{H s}+\mathbf{w}
$$

where $\mathbf{y}, \mathbf{w}$ and $\mathbf{s}$ denote the $n_{\mathrm{r}}$-dimensional received signal and AWGN sample vectors as well as the $m_{\mathrm{t}}$-dimensional transmitted signal vector, respectively. Furthermore, $\mathbf{H}$ represents a $\left(n_{\mathrm{r}} \times m_{\mathrm{t}}\right)$-dimensional matrix of subcarrier-related CTF coefficients. Note that for the sake of brevity we omit the OFDM subcarrier and symbol indices $k$ and $n$. where we omit the OFDM subcarrier and symbol indices $k$ and $n$, respectively.

As outlined in [1], the ML SDM detector provides an $m_{\mathrm{t}^{-}}$ antenna-based estimated signal vector candidate $\hat{\mathbf{s}}$, which maximizes the objective function defined as the conditional a posteriori probability function $\mathrm{P}\{\check{\mathbf{s}} \mid \mathbf{y}, \mathbf{H}\}$ over the set $\mathcal{M}^{\mathbb{1}_{\mathrm{t}}}$ of legit- 
imate solutions. More explicitly, we have

$$
\hat{\mathbf{s}}=\arg \max _{\check{\mathbf{s}} \in \mathcal{M} \mathbb{N}_{\mathrm{t}}} P\{\check{\mathbf{s}} \mid \mathbf{y}, \mathbf{H}\},
$$

where $\mathcal{M}^{\mathbb{1}_{\mathrm{t}}}$ is the set of all possible $m_{\mathrm{t}}$-dimensional candidate symbol vectors of the $m_{\mathrm{t}}$-antenna-based transmitted signal vector s. More specifically, we have

$$
\left.\mathcal{M}^{\mathbb{1}_{\mathrm{t}}}=\left\{\check{\mathbf{s}}=\left(\check{\int}_{\infty}, \cdots, \check{\int}_{\tilde{I}_{\mathrm{t}}}\right)^{\mathrm{T}} ; \check{\int}\right\rangle \in \mathcal{M}\right\} .
$$

Furthermore, it was shown in [1] that we have

$$
\mathrm{P}\{\check{\mathbf{s}} \mid \mathbf{y}, \mathbf{H}\}=A \exp \left[-\frac{1}{\sigma_{w}^{2}}\|\mathbf{y}-\mathbf{H} \check{\mathbf{s}}\|^{\mathbf{2}}\right],
$$

where $A$ is a constant, which is independent of any of the values $\left\{\check{s}_{i}\right\}_{i=1, \cdots, m_{\mathrm{t}}}$. Thus, it may be shown [1] that the probability maximization problem of Equation (2) is equivalent to the corresponding Euclidean distance minimization problem. Specifically, we have

$$
\hat{\mathbf{s}}=\arg \min _{\check{\mathbf{s}} \in \mathcal{M} \llbracket \mathrm{t}}\|\mathbf{y}-\mathbf{H} \check{\mathbf{s}}\|^{\mathbf{2}} .
$$

In our previous work [4] we have demonstrated that the ML solution of Equation (5) of a noisy linear problem described by Equation (1) is given by

$$
\hat{\mathbf{s}}=\arg \min _{\check{\mathbf{s}} \in \mathcal{M}^{\mathbb{I}_{t}}}\|\mathbf{U}(\check{\mathbf{s}}-\hat{\mathbf{x}})\|^{2},
$$

where the transmitted symbols $s$ satisfy the condition of $|s|^{2}=1$ and $\mathbf{U}$ is an upper-triangular matrix having positive real-valued elements on the main diagonal and satisfying

$$
\mathbf{U}^{\mathrm{H}} \mathbf{U}=\left(\mathbf{H}^{\mathrm{H}} \mathbf{H}+\sigma_{\mathbf{w}}^{\mathbf{2}} \mathbf{I}\right),
$$

while

$$
\hat{\mathbf{x}}=\left(\mathbf{H}^{\mathrm{H}} \mathbf{H}+\sigma_{\mathbf{w}}^{\mathbf{2}} \mathbf{I}\right)^{-\mathbf{1}} \mathbf{H}^{\mathrm{H}} \mathbf{y}
$$

is the unconstrained MMSE estimate of the transmitted signal vector $\mathbf{s}$.

Consequently, we define a new objective function $J(\check{\mathbf{s}})$ as follows [4]

$$
\begin{aligned}
J(\check{\mathbf{s}}) & =\|\mathbf{U}(\check{\mathbf{s}}-\hat{\mathbf{x}})\|^{2} \\
& =\sum_{i=1}^{m_{\mathrm{t}}}\left|\sum_{j=i}^{m_{\mathrm{t}}} u_{i j}\left(\check{s}_{j}-\hat{x}_{j}\right)\right|^{2}=\sum_{i=1}^{m_{\mathrm{t}}} \phi_{i}\left(\check{\mathbf{s}}_{i}\right),
\end{aligned}
$$

where $\phi_{i}\left(\check{\mathbf{s}}_{i}\right)$ are a positive real-valued sub-cost function.

Furthermore, let $J_{i}\left(\check{\mathbf{s}}_{i}\right)$ be a Cumulative Sub-Cost (CSC) function recursively defined as

$$
\begin{aligned}
J_{m_{\mathrm{t}}}\left(\check{s}_{m_{\mathrm{t}}}\right) & =\phi_{m_{\mathrm{t}}}\left(\check{s}_{m_{\mathrm{t}}}\right)=\left|u_{m_{\mathrm{t}} m_{\mathrm{t}}}\left(\check{s}_{m_{\mathrm{t}}}-\hat{x}_{m_{\mathrm{t}}}\right)\right|^{2} \\
J_{i}\left(\check{\mathbf{s}}_{i}\right) & =J_{i+1}\left(\check{\mathbf{s}}_{i+1}\right)+\phi_{i}\left(\check{\mathbf{s}}_{i}\right), \quad i=m_{\mathrm{t}}-1, \cdots, 1,
\end{aligned}
$$

where we define the candidate subvector as $\check{\mathbf{s}}_{i}=\left[\check{s}_{i}, \cdots, \check{s}_{m_{\mathrm{t}}}\right]$.

To elaborate a little further, the derivation of an expression for the evaluation of the soft-bit information associated with the bit estimates of the SDM detector's output characterized by Equation (2) is given in [1]. Here, we present a brief summary of the results deduced in [1].

The soft-bit value associated with the $m$ th bit of the QAM symbol transmitted from the $i$ th transmit antenna element is determined by the log-likelihood function defined in [10] as

$$
L_{i m}=\log \frac{\sum_{\check{\mathbf{s} \in \mathcal{M}_{i \mathbb{1}} ; \mathbb{t}}} \mathrm{P}\{\mathbf{y} \mid \check{\mathbf{s}}, \mathbf{H}\}}{\sum_{\check{\mathbf{s}} \in \mathcal{M}_{i \Uparrow \mathbb{t}}^{\prime ; \text { t }}} \mathrm{P}\{\mathbf{y} \mid \check{\mathbf{s}}, \mathbf{H}\}},
$$

where we define

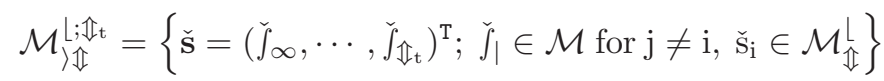

and $\mathcal{M}_{\mathbb{1}}^{L}$ denotes the specific subset of the entire set $\mathcal{M}$ of modulation constellation points, which comprises the bit value $b=\{0,1\}$ at the $m$ th bit position.

However, the direct calculation of the accumulate a posteriori conditional probabilities in the nominator and denominator of Equation (11) may have an excessive complexity in practice. Fortunately, as advocated in [1], the expression in Equation (11) can be closely approximated as follows

$$
L_{i m} \approx \log \frac{\mathrm{P}\left\{\mathbf{y} \mid \check{\mathbf{s}}_{\mathbf{i m}}^{\mathbf{1}}, \mathbf{H}\right\}}{\mathrm{P}\left\{\mathbf{y} \mid \check{\mathbf{s}}_{\mathbf{i m}}^{\mathbf{0}}, \mathbf{H}\right\}},
$$

where we define

$$
\check{\mathbf{s}}_{i m}^{b}=\arg \max _{\substack{\mathbf{s} \in \mathcal{M} \\ \text {; 隹 }}} \mathrm{P}\{\mathbf{y} \mid \check{\mathbf{s}}, \mathbf{H}\}, \quad b=0,1 .
$$

As suggested by the nature of Equation (13), the detection process employing the objective function determined by Equations (13) and (14) is usually referred to as the Logarithmic Maximum A Posteriori (Log-MAP) probability detector.

A practical version of the Log-MAP detector may be derived as follows. Substituting Equation (4) into (11) yields

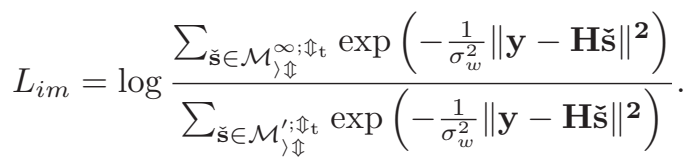

Note that Equation (15) involves two summations over $2^{b m_{\mathrm{t}}-1}$ exponential functions. This operation may potentially impose an excessive computational complexity for large values of $m_{\mathrm{t}}$ and/or $b$. However, as demonstrated in [1], the expression in (15) may be closely approximated by a substantially simpler expression, namely by

$$
L_{i m} \approx \frac{1}{\sigma_{w}^{2}}\left[\left\|\mathbf{y}-\mathbf{H} \check{s}_{\mathbf{i m}}^{\mathbf{0}}\right\|^{\mathbf{2}}-\left\|\mathbf{y}-\mathbf{H} \check{\mathbf{s}}_{\mathbf{i m}}^{\mathbf{1}}\right\|^{\mathbf{2}}\right],
$$

where we have

$$
\check{\mathbf{s}}_{i m}^{b}=\arg \min _{\check{\mathbf{s}} \in \mathcal{M}_{\zeta \Uparrow \mathbb{1}}^{i ; \pi_{\mathrm{t}}}}\|\mathbf{y}-\mathbf{H} \check{\mathbf{s}}\|^{\mathbf{2}}, \quad \mathbf{b}=\mathbf{0}, \mathbf{1},
$$

and again, $\mathcal{M}_{>\Uparrow \mathbb{1}}^{L ; \Uparrow t}$ denotes the specific subset of the entire set $\mathcal{M}^{\mathbb{1 t}_{\mathrm{t}}}$ of signal vector candidates associated with the modulation scheme employed, which comprises the bit value $b=\{0,1\}$ at the $m$ th bit position of the $i$ th signal vector component. 


\section{A. Bitwise approach}

As it was demonstrated in [7], the complex-valued system model of Equation (1) may be substituted by the corresponding real-valued bitwise system model, which may be expressed as follows

$$
\tilde{\mathbf{y}}=\tilde{\mathbf{H}} \mathbf{t}+\tilde{\mathbf{w}} .
$$

Additionally, in [4] we have shown that solving the detection problem of Equation (2) is equivalent to solving the following bitwise minimization problem

$$
\begin{aligned}
\hat{\mathbf{t}} & =\arg \max _{\check{\mathbf{t}} \in\{-1,1\}^{r}} P\{\check{\mathbf{t}} \mid \tilde{\mathbf{y}}, \tilde{\mathbf{H}}\} \\
& =\arg \min _{\check{\mathbf{t}} \in\{-1,1\}^{r}}\|\tilde{\mathbf{y}}-\tilde{\mathbf{H}} \check{\mathbf{t}}\|^{2},
\end{aligned}
$$

where $r$ denotes the number of columns of the real-valued bitwise channel matrix $\tilde{\mathbf{H}}$, which is equal to the product of the number of receive antennas $m_{\mathrm{t}}$ and the number of bits per QAM symbol $b$.

Consequently, the Log-MAP detector defined by Equations (16) and (17) may be applied for obtaining the soft-bit information associated with the bitwise OHRSA ML SDM detector of Equation (19). Substituting the bitwise system model of Equation (18) into (16) and (17) yields

$$
L_{i} \approx \frac{1}{\sigma_{w}^{2}}\left[\left\|\mathbf{y}-\tilde{\mathbf{H}} \check{\mathbf{t}}_{\mathbf{i} ; \min }^{0}\right\|^{2}-\left\|\mathbf{y}-\tilde{\mathbf{H}} \check{\mathbf{t}}_{\mathbf{i} ; \min }^{1}\right\|^{2}\right],
$$

where we have

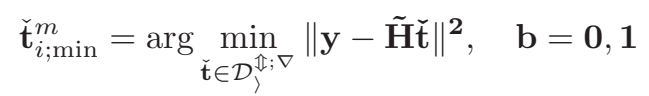

and $\mathcal{D}_{\rangle}^{\mathbb{1} ; \nabla}$ denotes the subset of the entire set $\mathcal{D}^{\nabla}$ of $(r=$ $\left.m_{\mathrm{t}} \log _{2} M\right)$-dimensional bitwise vectors, which comprise the binary value $\check{t}_{i}=d_{m}=\{-1,1\}$ at the $i$ th bit position.

Furthermore, substituting the bitwise objective function of Equation (16) into (20) yields

$$
\begin{aligned}
L_{i} & \approx \frac{1}{\sigma_{w}^{2}}\left[J\left(\check{\mathbf{t}}_{i ; \min }^{0}\right)+\phi-J\left(\check{\mathbf{t}}_{i ; \min }^{1}\right)-\phi\right] \\
& =\frac{1}{\sigma_{w}^{2}}\left[J\left(\check{\mathbf{t}}_{i ; \min }^{0}\right)-J\left(\check{\mathbf{t}}_{i \text { min }}^{1}\right)\right],
\end{aligned}
$$

where $\check{\mathbf{t}}_{i ; \text { min }}^{m}$ and the corresponding cost function value $J\left(\check{\mathbf{t}}_{i: \min }^{m}\right)$ may be obtained by applying the constrained OHRSAaided ML detection method derived in [4].

Consequently, the evaluation of the bitwise Log-MAP estimates of the transmitted bitwise signal vector $\mathbf{t}$ involves repetitive evaluation of $2 r$ constrained ML estimates $\check{\mathbf{t}}_{i ; \min }^{m}$ along with the associated $2 r$ values of the objective function $J\left(\check{\mathbf{t}}_{i ; \text { min }}^{m}\right)$.

The operation of the OHRSA-aided Log-MAP detector in the scenario of using BPSK and employing $m_{\mathrm{t}}=n_{\mathrm{r}}=3$ transmit and receive antennas is illustrated in Figure 1. Specifically, the repetitive search process generates $2 \cdot 3=6$ search trees associated with obtaining the ML estimates of the bitwise signal vector $\mathbf{t}$ constrained by bit-component value $\check{t}_{i}=d_{m}, i=$ $1,2,3, d_{m}=-1,1$. The resultant six search trees obtained by applying the OHRSA-Log-MAP detection method are depicted in Figures 1 ( $\mathrm{a}-\mathrm{f})$.

Clearly, the repetitive nature of the OHRSA-Log-MAP search process exemplified by Figure 1 imposes a substantial increase in the associated computational complexity. Hence, in the next section we derive an OHRSA-aided approximate LogMAP method, which is capable of approaching the optimum Log-MAP performance, while avoiding the repetitive evaluation and therefore imposes considerably reduced complexity requirements.

\section{Soft-Output Optimized Hierarchy-AIDED APPROXIMATE LOG-MAP SDM DETECTION}

As we may conclude from a closer analysis of the search-tree diagrams depicted in Figures $1(\mathrm{a}-\mathrm{f})$, a substantial amount of information concerning the reliability of possible values of the transmitted bit-representations $t_{i}$ of the $m_{\mathrm{t}}$-antenna-based symbols becomes available in the process of determining the ML estimate $\hat{\mathbf{t}}$ of Equation (19). Consequently, it is beneficial to exploit this information and hence to avoid the extra complexity imposed by the repetitive search involved in the Log-MAP method derived in Section II. In order to derive an appropriate soft-output RSA we make the following proposition.

Proposition 1: Let $\mathbf{y}, \tilde{\mathbf{H}}$ and $\mathbf{t}$ be the quantities involved in the system model of Equation (19), namely the received signal vector, the bitwise channel matrix and the transmitted bit-based signal vector, respectively. Furthermore, let $J_{i}\left(\check{\mathbf{t}}_{i}\right)$ be a CSC function defined by Equation (10). Then the a posteriori probability associated with the candidate value $\check{t}_{i}=\{-1,1\}$ of the $i$ th component of the bit-based signal vector $\tilde{\mathbf{t}}$ may be approximated as

$$
\mathrm{P}\left\{\check{t}_{i} \mid \mathbf{y}, \tilde{\mathbf{H}}\right\} \approx A_{i} \sum_{\check{\mathbf{t}}_{i+1} \in \mathcal{D} \nabla->} \exp -\frac{1}{\sigma_{w}^{2}} J_{i}\left(\left[\check{t}_{i}, \check{\mathbf{t}}_{i+1}^{\mathrm{T}}\right]^{\mathrm{T}}\right),
$$

where $A_{i}$ is a scalar independent of any of the values of $\left\{\check{t}_{i}\right\}_{i=1, \cdots, r}$.

Note 1. A particularly interesting implication of the proposition outlined above is that the a posteriori probability associated with the given value of the bit $t_{i}$ may be approximately determined, regardless of the values of the bits situated in the hierarchy of the tree-diagrams depicted in Figures $1(\mathrm{a}-\mathrm{f})$ at levels lower than level $i$. In other words, the approximate a posteriori probability value $\mathrm{P}\left\{\check{t}_{i} \mid \tilde{\mathbf{H}}, \mathbf{y}\right\}$ may be evaluated independently of any of the values $\left\{\check{t}_{j}\right\}_{j=1, \cdots, i-1}$.

Note 2. Another important implication of Proposition 1, which may be directly inferred from Note 1 , is that given the appropriate sorting of the bitwise channel matrix $\tilde{\mathbf{H}}$, the process of SDM detection may be reduced to detecting the transmitted symbol components $t_{i}, \cdots, t_{m_{\mathrm{t}}}$ associated with the good channel conditions. As a consequence of Note 1, reducing the scope of the SDM detection process will not substantially affect the detection quality of the reliable signal components. This property is particularly interesting in the scenario of heavily overloaded multi-user SDMA systems, where we may be interested in detecting only the high-power users' signals at each particular base-station, while suppressing the interference imposed by 


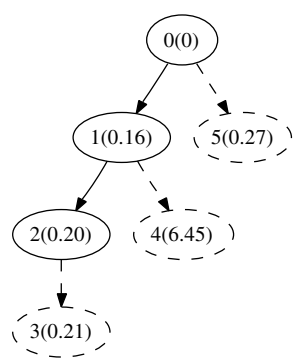

(a)

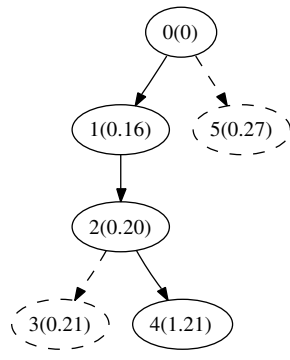

(d)

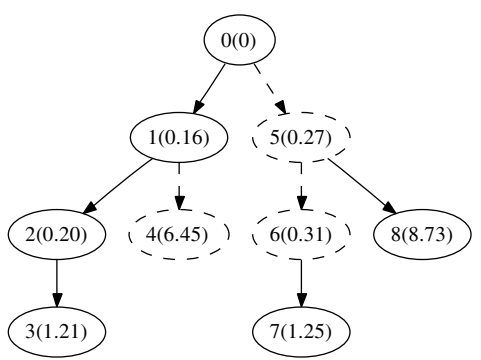

(b)

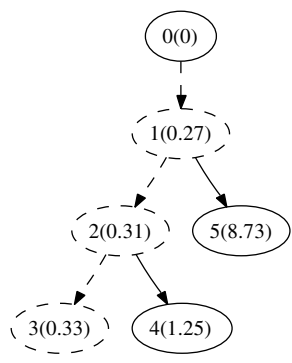

(e)

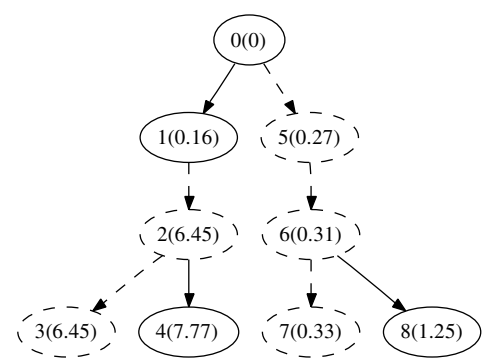

(c)

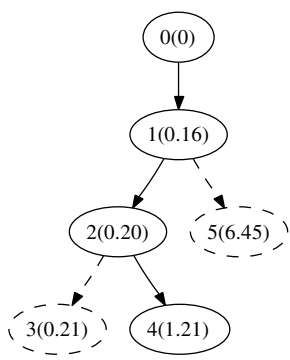

(f)

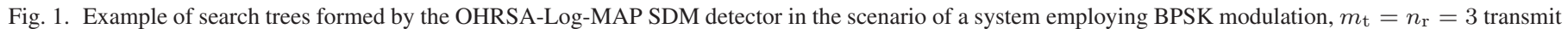
and receive antennas and encountering average SNRs of $10 \mathrm{~dB}$. The labels indicate the order of visitation, as well as the corresponding value $J_{i}\left(\check{\mathbf{t}}_{i}\right)$ of the CSC function, as seen in the brackets.

the low-power users without attempting to actually detect their signals.

The major justification of the validity of Proposition 1 accrues from the high efficiency of the detection method derived.

Let us now define the $(r \times 2)$-dimensional Bitwise Cumulate Cost (BWCC) function matrix $\hat{\mathbf{J}}$ having elements as follows

$$
\hat{J}_{i m}=-\operatorname{JacLog}\left(\left\{-J_{i}\left(d_{m}, \check{\mathbf{t}}_{i+1}^{\mathrm{T}}\right)\right\}_{\check{\mathbf{t}}_{i+1} \in \mathcal{D} \mathbb{\Perp}_{\mathrm{t}-\rangle}}\right)
$$

for $i=1, \cdots, r, m=0,1$, where $\operatorname{JacLog}(\cdot)$ denotes the Jacobian logarithm function defined as

$$
\operatorname{JacLog}(a, b, \cdots, c)=\log \left(e^{a}+e^{b}+\cdots+e^{c}\right) .
$$

When using the BWCC function of Equation (24), the a posteriori probability function of Equation (23) may be expressed as

$$
\mathrm{P}\left\{\check{t}_{i}=d_{m} \mid \mathbf{y}, \tilde{\mathbf{H}}\right\}=A_{i} \exp \left[-\frac{1}{\sigma_{w}^{2}} \hat{J}_{i m}\left(\left[d_{m}, \check{\mathbf{t}}_{i+1}^{\mathrm{T}}\right]^{\mathrm{T}}\right)\right]
$$

for $i=1, \cdots, r, m=0,1$.

A simple expression deduced for the evaluation of the soft-bit information vector derived in Section II is generated by substituting the conditional probability function of Equation (26) into (13), which yields

$$
\begin{aligned}
L_{i} & =\log \frac{\mathrm{P}\left\{\check{t}_{i}=d_{0} \mid \mathbf{y}, \tilde{\mathbf{H}}\right\}}{\mathrm{P}\left\{\check{t}_{i}=d_{1} \mid \mathbf{y}, \tilde{\mathbf{H}}\right\}} \\
& =\frac{1}{\sigma_{w}^{2}}\left[\hat{J}_{i 0}-\hat{J}_{i 1}\right], i=1, \cdots, r .
\end{aligned}
$$

Observe that the constant scalar $A_{i}$ of Equation (23) appears both in the nominator and denominator of Equation (27) and therefore may be discarded. Equation (28) may be also expressed in a vectorial form as

$$
\mathbf{L}=\frac{\mathbf{1}}{\sigma_{\mathbf{w}}^{\mathbf{2}}}\left[(\hat{\mathbf{J}})_{\mathbf{0}}-(\hat{\mathbf{J}})_{\mathbf{1}}\right]
$$

where, as before, $(\hat{\mathbf{J}})_{m}$ denotes the $m$ th column of the matrix $\hat{\mathbf{J}}$ having elements defined by Equation (24).

Finally, by supplementing Equations (24) and (29) into the OHRSA-Log-MAP method of Section II, we arrive at an approximate OHRSA-Log-MAP SDM detector, which avoids the repetitive search required by the OHRSA-Log-MAP SDM detector.

The search tree diagram generated by applying the approximate OHRSA-Log-MAP SDM detector described above in the scenario of using BPSK and employing $m_{\mathrm{t}}=n_{\mathrm{r}}=3$ transmit and receive antennas is depicted in Figure 2(a). A close analysis of the search tree-diagram of Figure 2(a) reveals that the information collected by the search process of the modified OHRSA-ML method may be insufficient for the reliable evaluation of the soft-bit information associated with some of the bits of the transmitted bitwise signal vector $\mathbf{t}$. Consequently, a certain performance degradation may be expected. In order to mitigate this undesirable effect, we introduce the additional parameter $\rho$, which we refer to as the search radius factor. More specifically, the parameter $\rho$ allows us to control the rate of convergence for the OHRSA-ML search process derived in [4] and affects the cut-off value of a CSC function, which limits the passage of the recursive search process through low likelihood search branches having the CSC function value $J_{i}\left(\check{\mathbf{t}}_{i}\right)$ in excess of $\rho J_{\min }$, as opposed to $J_{\min }$. The search trees formed by the execution of the modified OHRSA-ML method in the scenarios of setting $\rho=1.3$ and $\rho=2.0$ are depicted in Figures 2 (b) and (c), respectively. 


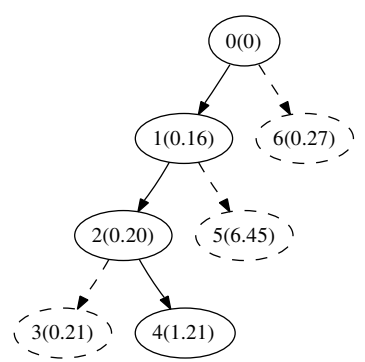

(a)

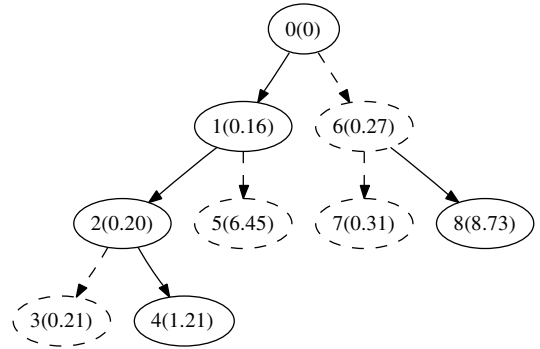

(b)

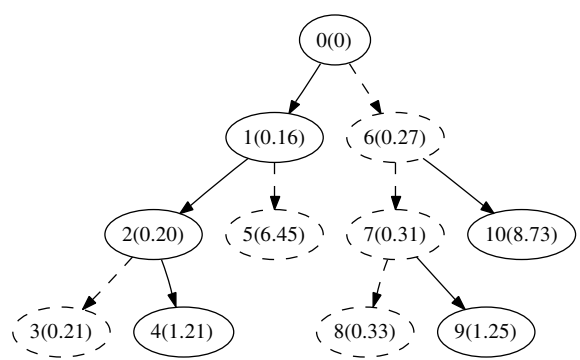

(c)

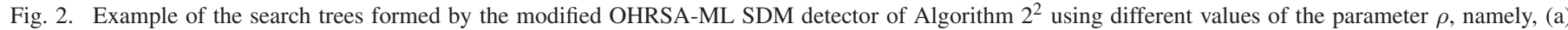
$\rho=1.0$, (b) 1.3 and (c) 2.0. We consider a system employing BPSK modulation, $m_{\mathrm{t}}=n_{\mathrm{r}}=3$ transmit and receive antennas and encountering an average SNR of $10 \mathrm{~dB}$. The labels indicate the order of evaluation, as well as the corresponding value $J_{i}\left(\check{\mathbf{s}}_{i}\right)$ of the CSC function, as seen in the brackets.

Observe that the higher the value of $\rho$, the slower the convergence of the search process is. The suitable value of the global search radius factor $\rho$ may be found empirically and its exact value within a sensible range has in fact little effect on the attainable performance and the associated complexity of the proposed algorithm. More specifically, we have found that the range of values spanning the interval $1.2<\rho<1.7$ is appropriate and a meritorious value for the majority of system configurations is $\rho=1.3$.

The resultant OHRSA-aided approximate Log-MAP SDM detector, which we refer to as the Soft-output OPtimized HIErarchy (SOPHIE) SDM detector is summarized in Algorithm 1.

\section{Performance Analysis}

TABLE I

SYSTEM PARAMETERS.

\begin{tabular}{|l|c|c|}
\hline Parameter & OFDM & MC-CDMA \\
\hline \hline Channel bandwidth & \multicolumn{2}{|c|}{$800 \mathrm{kHz}$} \\
\hline Number of carriers $K$ & \multicolumn{2}{|c|}{128} \\
\hline Symbol duration $T$ & \multicolumn{2}{|c|}{$160 \mu \mathrm{s}$} \\
\hline Max. delay spread $\tau_{\max }$ & \multicolumn{2}{|c|}{$40 \mu \mathrm{s}$} \\
\hline Channel interleaver & $\begin{array}{c}\text { WCDMA [9] } \\
248 \text { bit }\end{array}$ \\
\hline Modulation & \multicolumn{2}{|c|}{ QPSK } \\
\hline Spreading scheme & \multicolumn{2}{|c|}{ WH } \\
\hline $\begin{array}{l}\text { FEC } \\
\text { component codes } \\
\text { code interleaver }\end{array}$ & $\begin{array}{c}\text { Turbo code [8] rate 1/2 } \\
\text { RSC, K=3(7,5) }\end{array}$ \\
\hline
\end{tabular}

Our simulations were performed in the base-band frequency domain. The OFDM system considered utilises 128 QAMmodulated orthogonal subcarriers. For forward error correction (FEC) we use $\frac{1}{2}$-rate turbo coding [8] employing two constraintlength $K=3$ Recursive Systematic Convolutional (RSC) component codes and the standard 124-bit WCDMA UMTS turbo code interleaver of [9]. The octally represented RCS generator polynomials of $(7,5)$ were used. We assume a 9-tap CIR Rayleigh-fading multipath channel and stipulate the assumption of perfect channel knowledge, where the knowledge of the frequency-domain subcarrier-related coefficients $H[n, k]$ is

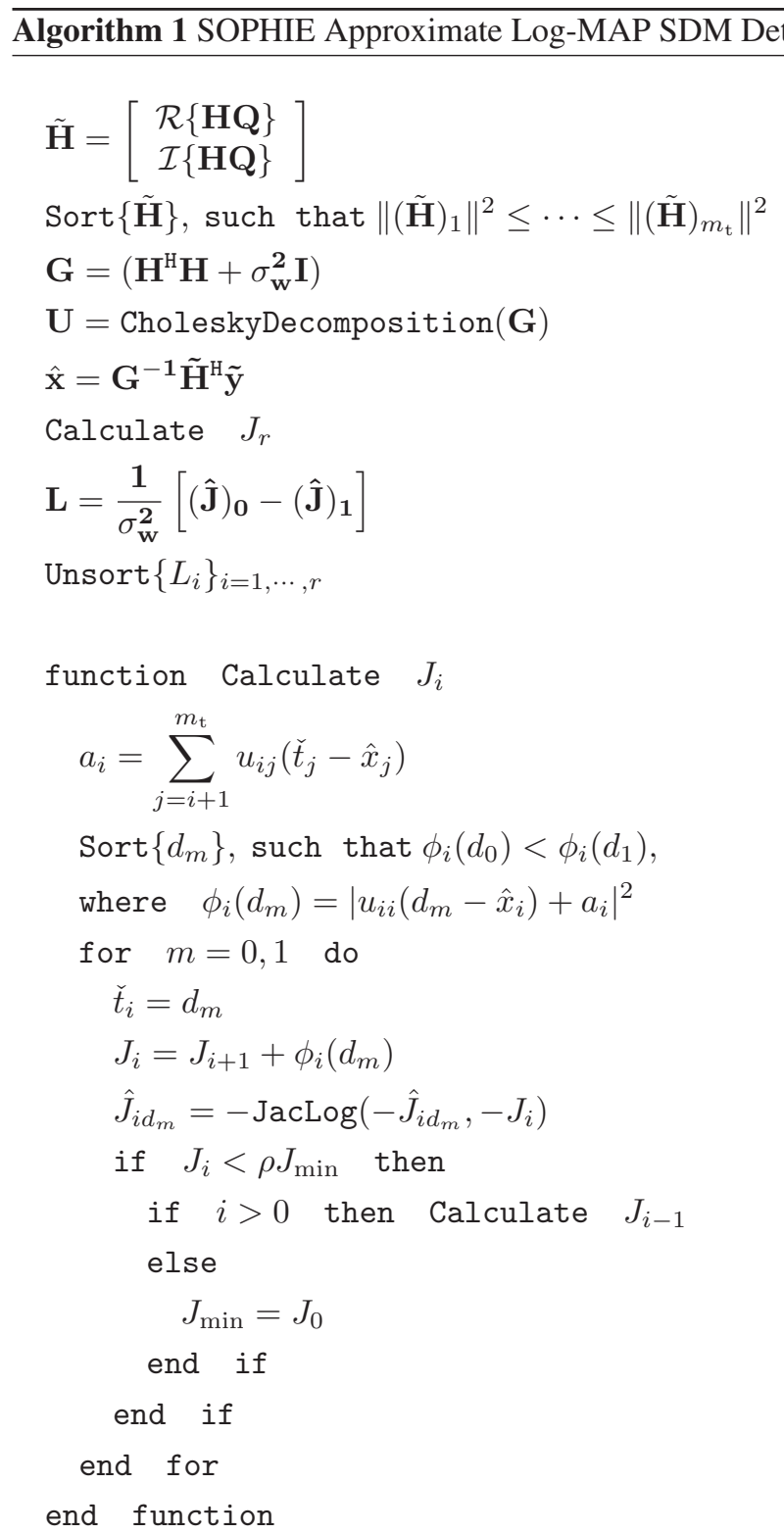

deemed to be available in the receiver.

Figure 3 characterizes the achievable BER performance of 


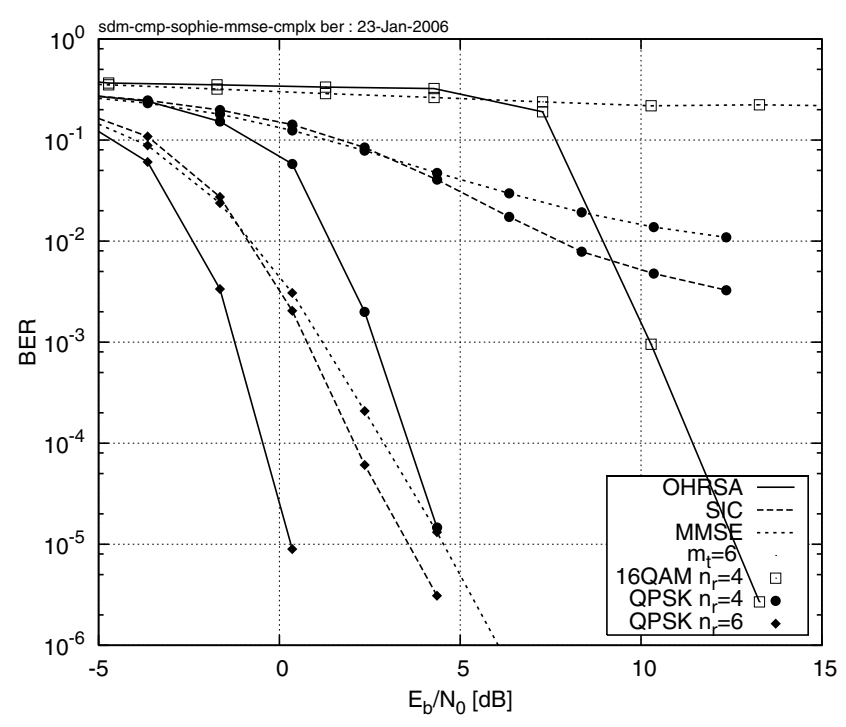

Fig. 3. Bit Error Rate exhibited by the rate- $\frac{1}{2}$ turbo-coded SDM-OFDM system employing the SOPHIE SDM detector of Algorithm 1. The abscissa represents the average $E_{b} / N_{0}$. The OFDM system parameters are summarized in Table I.

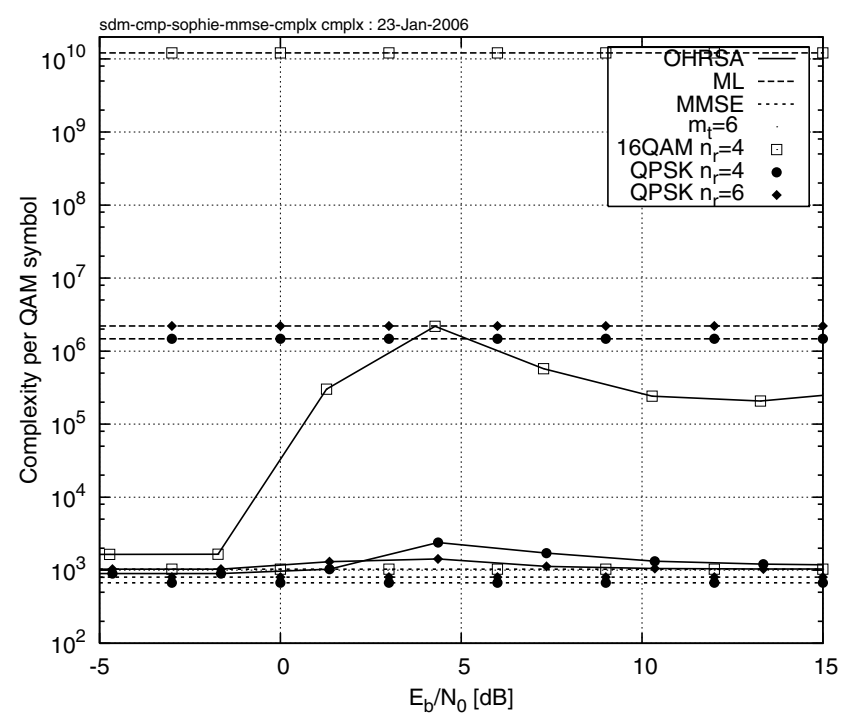

Fig. 4. Computational complexity, quantified in terms of the total number of additions and multiplications, exhibited by the rate- $\frac{1}{2}$ turbo-coded SDMOFDM system employing the SOPHIE SDM detector of Algorithm 1. The abscissa represents the average $E_{b} / N_{0}$. The OFDM system parameters are summarized in Table I.

the MIMO-OFDM system employing the SOPHIE detector proposed, as well as that of a number of other popular detection methods, such as the MMSE and SIC detectors. We can see that as opposed to the MMSE and SIC detectors, the proposed SOPHIE detector performs equally well both in fully-loaded as well as in overloaded scenarios, where the number of transmit antennas exceeds that of the receive antennas and thus we have $m_{\mathrm{t}}>n_{\mathrm{r}}$. Furthermore, the SOPHIE method exhibits an equally good performance, when employed in the overloaded 16-QAMmodulated MIMO-OFDM system.

On the other hand, Figure 4 illustrates the corresponding computational complexity quantified in terms of the total number of real addition and multiplication operations per detected QAM symbol. Observe that the complexity exhibited by the SOPHIE method in the QPSK scenario is only slightly higher than that exhibited by the MMSE detector. Moreover, the complexity exhibited by the SOPHIE detector in the overloaded 16-QAM scenario is almost five orders of magnitude lower than that exhibited by the exhaustive ML detector, while their BER performance is expected to be similar. We note however that the BER performance of the ML detector is not shown since it would impose a more than $10^{4}$-times higher complexity.

\section{CONClusion}

In this paper we proposed a so-called SOPHIE SDM detection method, which may be regarded as an advanced extension of the CSD method. We have shown that the SOPHIE SDM detector proposed closely approaches the optimum performance of the Log-MAP detector while imposing a dramatically lower computational complexity. In particular in the scenario of employing QPSK the complexity associated with both SOPHIE and softoutput linear MMSE detectors may be shown to be nearly similar. Moreover, the method is readily applicable to OFDM systems employing high-throughput modulation schemes, such as $M$-QAM. Particularly, in the ( $M \geq 16)$-QAM single-antenna scenario the SOPHIE detector imposes substantially lower complexity than its soft-output MMSE counterpart, which renders it an attractive solution even in the conventional SISO communication systems.

Additionally, the SOPHIE detector may be employed in the over-loaded scenario, where the number of transmit antenna elements exceeds that of the receive antenna elements, while the associated computational complexity increases only moderately even in heavily overloaded scenarios and is almost independent of the number of receive antennas. The method proposed maintains the near-optimal performance of the Log-MAP SDM detector even in the heavily over-loaded scenarios.

\section{REFERENCES}

[1] L. Hanzo, M. Münster, B. J. Choi, and T. Keller. OFDM and MC-CDMA for Broadband Multi-User Communications, WLANs and Broadcasting. John Wiley and IEEE Press, 2003. 992 pages.

[2] L. Hanzo, L-L. Yang, E-L. Kuan, and K. Yen. Single- and Multi-Carrier DS-CDMA. John Wiley and IEEE Press, 2003. 1080 pages.

[3] A. Goldsmith, S. A. Jafar, N. Jindal, and S. Vishwanath. Capacity limits of MIMO channels. IEEE Journal on Selected Areas in Communications, 21(5):684-702, June 2003

[4] J. Akhtman and L. Hanzo. Reduced-complexity maximum-likelihood detection in multiple-antenna-aided multicarrier systems. In Proceedings of the 5th Int'l Workshop on Multi-Carrier Spread Spectrum, Oberpfaffenhofen, Germany, 14-16 September 2005. to appear

[5] B. M. Hochwald and S. ten Brink. Achieving near-capacity on a multipleantenna channel. IEEE Transactions on Communications, 51(3):389-399, March 2003.

[6] D. Pham, K. R. Pattipati, P. K. Willet, and J. Luo. An improved complex sphere decoder for V-BLAST Systems. IEEE Signal Processing Letters, 11(9):748-751, September 2004.

[7] T. Cui and C. Tellambura. An efficient generalized sphere decoder for rank-deficient mimo systems. IEEE Communications Letters, 9(5):423425, 2005.

[8] L. Hanzo, T. H. Liew, and B. L. Yeap. Turbo Coding, Turbo Equalisation and Space-Time Coding. John Wiley and IEEE Press, Chichester, UK; Piscataway, NJ, USA, 2002. 766 pages. (For detailed contents, please refer to http://www-mobile.ecs.soton.ac.uk.).

[9] H. Holma and A. Toskala, editors. WCDMA for UMTS : Radio Access for Third Generation Mobile Communications. John Wiley and Sons, Ltd., 2000.

[10] T.K. Moon and W.C. Stirling. Mathematical Methods and Algorithms for Signal Processing. Prentice Hall, 2000. 